\title{
Black Phosphorus Quantum Dots in Aqueous Ethylene Glycol for Macroscale Superlubricity
}

Xiaoyong Ren, Xiao Yang, Guoxin Xie*, Jianbin Luo*

State Key Laboratory of Tribology, Department of Mechanical Engineering, Tsinghua University, Beijing

100084, China

*Corresponding author,E-mail:xgx2014@,tsinghua.edu.cn; luojb@tsinghua.edu.cn

Figure S1. The characterization of the initial RP powder and the prepared BP powder: typical SEM images of (a) the initial RP powder and (b) the prepared BP powder; The XRD patterns (c) and Raman spectra (d) of the initial RP powder and prepared BP powder.

Figure S2. a) Image of as-prepared BPQDs ethanol suspension; b) images of BPQDs-EG suspensions with various concentrations of BPQDs: (1) $30 \mathrm{ppm}$, (2) $90 \mathrm{ppm}$, (3) $150 \mathrm{ppm}$, and c) the corresponding images after two weeks; d) images of BPQDs-PA suspensions with different kinds of polyhydric alcohols: (1) BPQDs-EG, (4) BPQDs-PG, (5)BPQDs-BG), and e) the corresponding images after two weeks.

Figure S3. a) XPS full spectra of the BP powders and BPQDs; b) XPS spectrum of O 1s peaks of the BPQDs; c) XPS spectrum of P 2p peaks of the BP powder; and d) XPS spectrum of O 1s peaks of the BP powders.

Figure S4. TEM image of the morphology of EG after drying on the ultrathin carbon film.

Figure S5. Variation curves of the COFs of the BPQDs-EG $\mathrm{Eq}_{\mathrm{aq}}$ suspensions at different sliding speeds at the normal load of $3 \mathrm{~N}$.

Figure S6. (a) The concentration of BPQDs- $\mathrm{EG}_{\mathrm{aq}}$ suspension measured as a function of time during the friction test at a load of $3 \mathrm{~N}$ and a speed of $100 \mathrm{~mm} / \mathrm{s}$; (b) the viscosity as a function of the concentrations of BPQDs-EGaq suspensions at $25{ }^{\circ} \mathrm{C}$.

Figure S7. XPS full spectra in the worn regions on the ball and disc surfaces. 


\section{S1. Characterizations of the prepared black phosphorus (BP) powders}
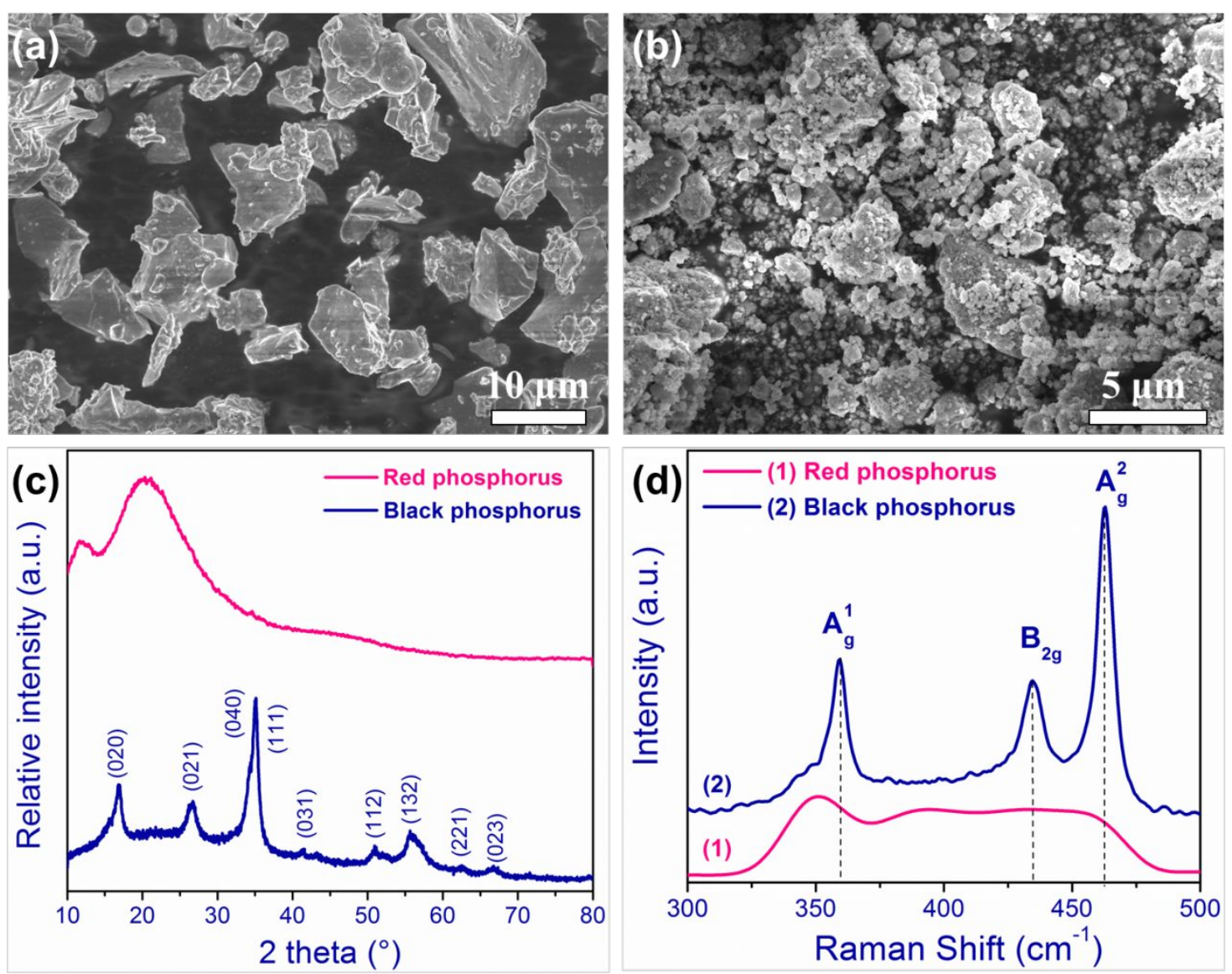

Figure S1. The characterization of the initial RP powder and the prepared BP powder: typical SEM images of (a) the initial RP powder and (b) the prepared BP powder; The XRD patterns (c) and Raman spectra (d) of the initial RP powder and prepared BP powder.

The BP powders were prepared through the high energy ball milling technique. Red phosphorus (RP) with a purity $>99 \%$ (RP, Aladdin Ltd) was used as the starting material. The ball milling process was carried at a speed of $800 \mathrm{rpm}$ for $20 \mathrm{~h}$ with stainless steel balls in a steel jar. Figure S1 depicts the typical SEM images of the raw RP powder and as-prepared BP powder. It can be seen that the shape of the raw RP particle was irregular and the size ranged from several to tens of micrometer. The size of the prepared BP was much smaller than that of the RP powder, and the particle shape was still irregular. Figure S1c,d show the XRD patterns and the Raman spectra of the prepared BP powder and the raw RP powder. After the long-time ball milling process, the diffraction peaks of RP disappeared, and instead distinct diffraction peaks appeared at $2 \theta=16,26,35$ and $56^{\circ}$, being consistent with those of the standard orthorhombic BP (JCPDS No. 76-1957). The Raman spectra analysis also confirmed the successful transformation from RP to BP. The peaks of BP 
at 361,438 and $466 \mathrm{~cm}^{-1}$ were attributed to the $\mathrm{A}_{\mathrm{g}}{ }^{1}, \mathrm{~B}_{2 \mathrm{~g}}$ and $\mathrm{A}_{\mathrm{g}}{ }^{2}$ phonon modes for the bulk BP. The characterizations of the BP confirmed the successful preparation of the BP powder by the high energy ball milling technique.

\section{S2. Preparation of BPQDs-EG, BPQDs-PG and BPQDs-BG suspensions.}

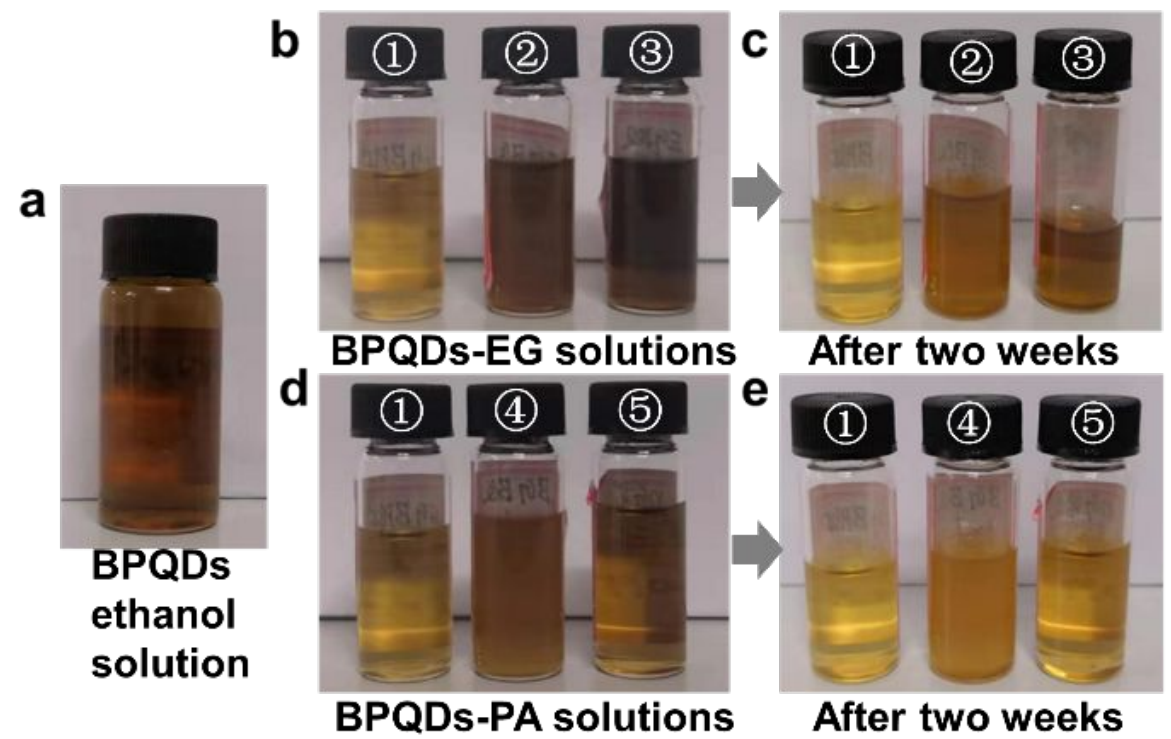

Figure S2. a) Image of as-prepared BPQDs ethanol suspension; b) images of BPQDs-EG suspensions with various concentrations of BPQDs: (1) $30 \mu \mathrm{g} / \mathrm{mL}$, (2) $90 \mu \mathrm{g} / \mathrm{mL}$, (3) $150 \mu \mathrm{g} / \mathrm{mL}$, and c) the corresponding images after two weeks; d) images of BPQDs-PA suspensions with different kinds of polyhydric alcohols: (1) BPQDs-EG, (4) BPQDs-PG, (5) BPQDs-BG), and e) the corresponding images after two weeks.

Figure S2 depicts the optical images of the BPQDs ethanol and BPQDs-PA suspensions to reveal the colors of the prepared suspensions. It can be seen that the BPQDs ethanol suspension was a brown translucent liquid. The solvents were changed to PA by mixing the BPQDs ethanol suspension with EG, PG and BG, respectively, and then ethanol was removed by evaporation. Figure S2b,d depict the optical images of BPQDs-EG, BPQDs-PG and BPQDs-BG suspensions. The colors of these suspensions were brown and translucent and the color became dark as the concentration increased. The color of the BPQDs-PA suspensions only changed slightly even after two weeks, as shown in Figure S2c,e, revealing the stability of BPQDs was improved with the protection of PA. 


\section{S3. Characterization of BPQDs.}
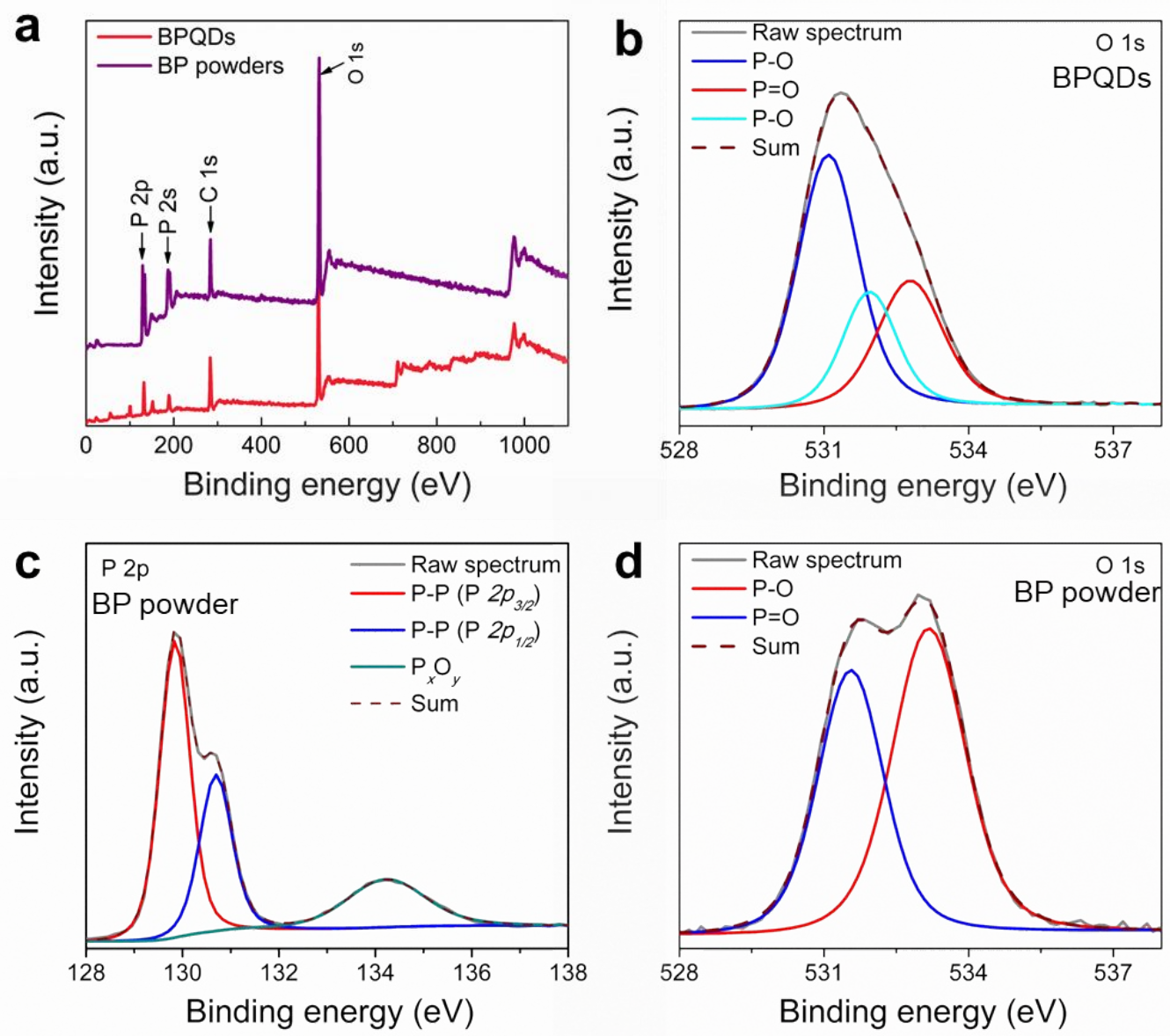

Figure S3. a) XPS full spectra of the BP powders and BPQDs; b) XPS spectrum of O 1s peaks of the BPQDs; c) XPS spectrum of $\mathrm{P} 2 \mathrm{p}$ peaks of the BP powder; and d) XPS spectrum of O 1s peaks of the BP powders.

Figure S3a depicts the XPS full spectra of the BPQDs and the BP powder, and P 2p peak and O 1s peak can be observed on both BP and BPQDs spectra.

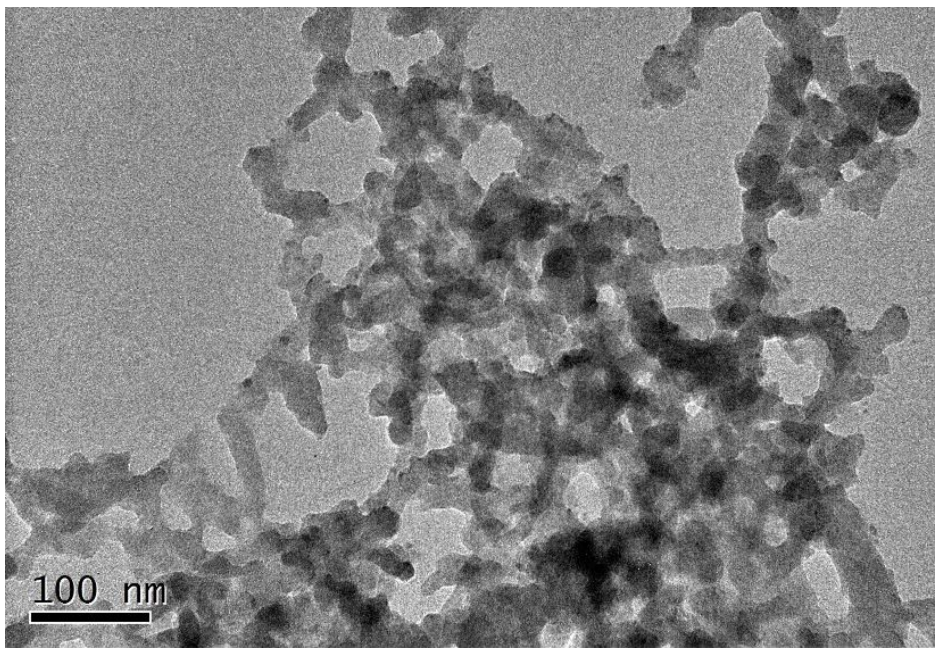

Figure S4. TEM image of the morphology of EG after drying on the ultrathin carbon film. 


\section{S4. Friction tests of BPQDs-EG ${ }_{\text {aq }}$.}

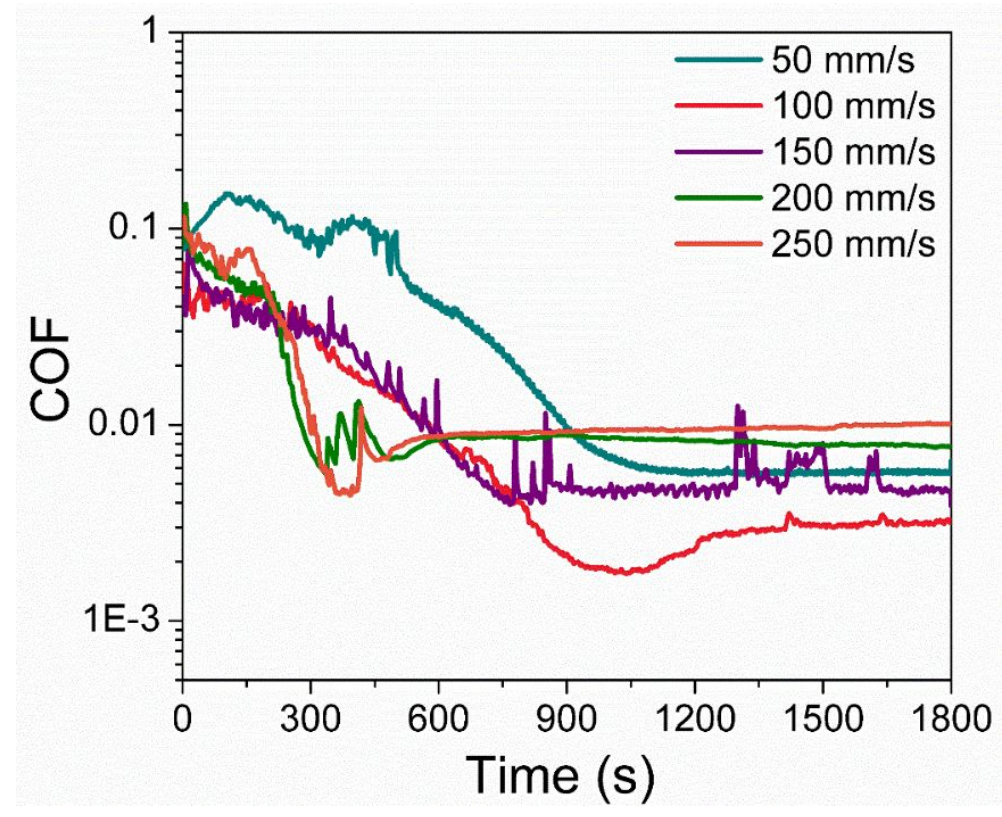

Figure S5. The variation of COFs of the BPQDs- $\mathrm{EG}_{\mathrm{aq}}$ suspensions at different sliding speeds under $3 \mathrm{~N}$.
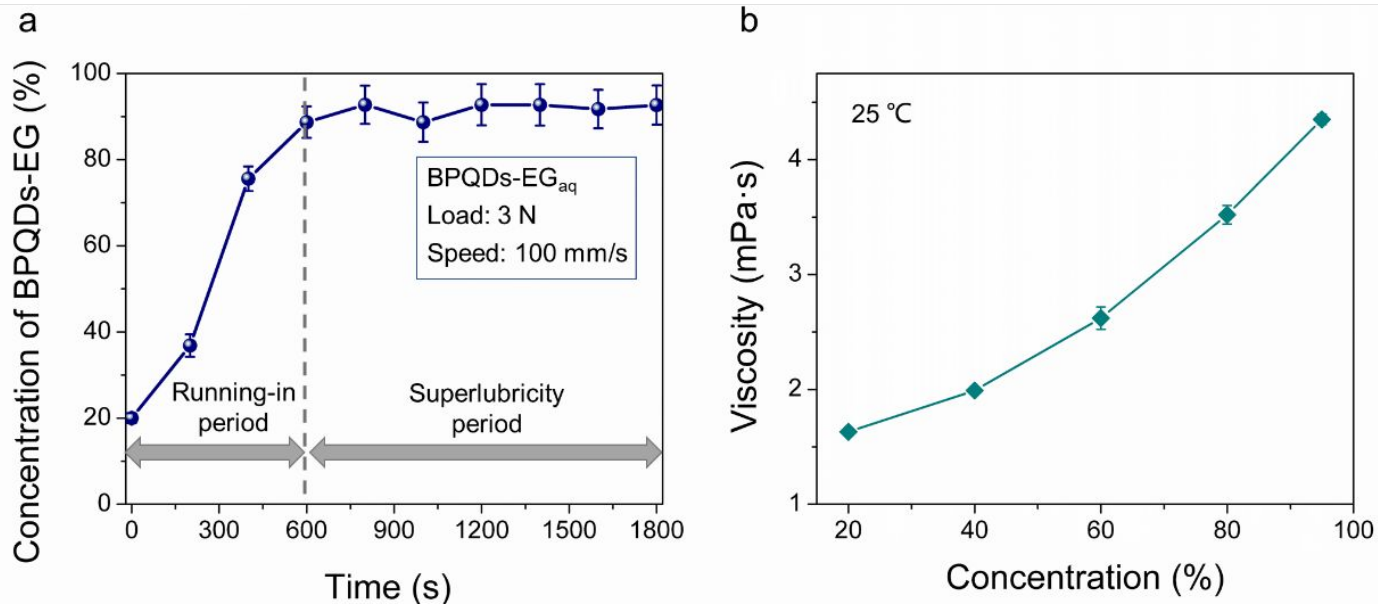

Figure S6. (a) The concentration of BPQDs- $\mathrm{EG}_{\mathrm{aq}}$ suspension measured as a function of time during the friction test at a load of $3 \mathrm{~N}$ and a speed of $100 \mathrm{~mm} / \mathrm{s}$; (b) the viscosity as a function of the concentrations of BPQDs-EGaq suspensions at $25{ }^{\circ} \mathrm{C}$.

\section{S5. Characterization of the wear surface after friction tests.}




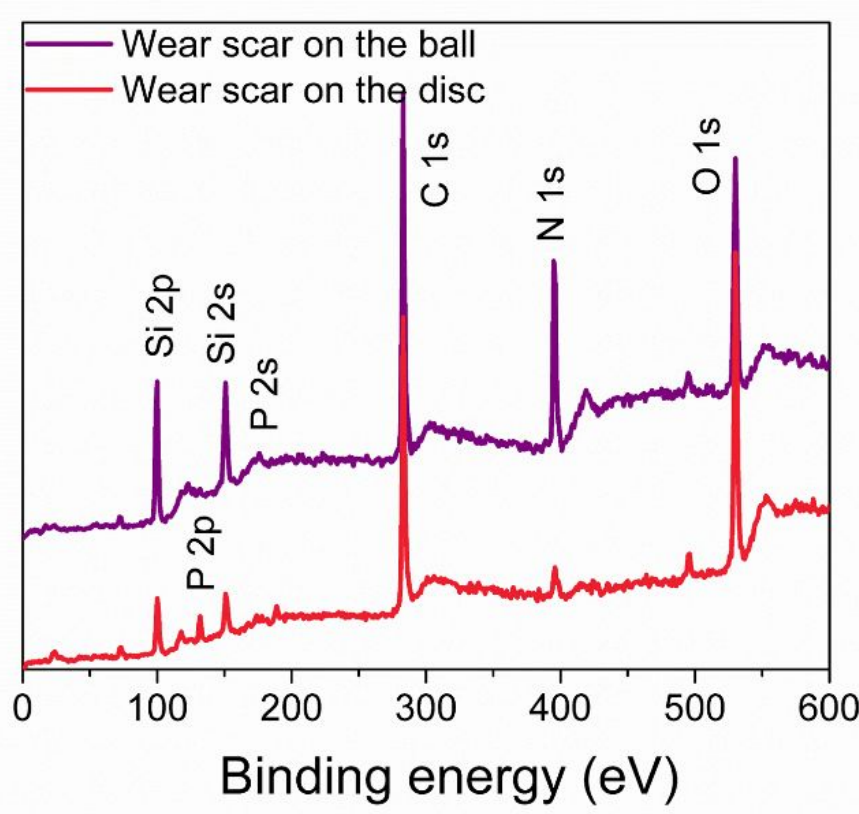

Figure S7. XPS full spectra in the worn regions on the ball and disc surfaces. 